\title{
Research on Combat Operation of Airborne Jamming Pods
}

\author{
Yulin Yang, \\ Department of Aeronautical Electronic Engineering \\ The First Aeronautical Institute of Air Force \\ Xinyang, China \\ e-mail: yanyline@163.com
}

\author{
Lijuan Qiu \\ Department of Aeronautical Electronic Engineering \\ The First Aeronautical Institute of Air Force \\ Xinyang, China
}

\begin{abstract}
The aim of this study was to evaluate the jamming efficacy of the airborne jamming pods, so we analyzed the combat operation requirements of them, simulated the jamming ability of the pods to jam the ground-to-air defense radar when the airborne fighting at different tactical backgrounds. The simulation results showed that the jamming pods can effectively reduce the detection distance of the ground-to-air defense radar and improve the aircraft's probability of successful penetration when fighting at low-altitude or extreme low-altitude.
\end{abstract}

Keywords-collaborative combat; jamming efficacy; jamming pod; blanket jamming;electronic countermeasure $(E W)$

\section{INTRODUCTION}

Airborne jamming pods are airborne EW equipment which mounted on the carrier aircraft, to complement or enhance the self-defense EW ability of the aircraft, carry out air-to-air or air-to-ground electronic jamming missions, improve the aircraft's survivability and combatting effectiveness[1].

\section{COMBAT REQUIREMENTS OF THE JAMMING PODS}

The combat mission of the jamming pods is carrying out electronic jamming. According to the characteristics of the battlefield electromagnetic environment, the jammed object requirements of the pods are the continuous wave $(\mathrm{CW})$ radars, conventional pulse radars, pulse Doppler(PD) radars, pulse compression(PC) radars, frequency agile radars, phased array radars, missile's active homing guidance radars, etc.[2]; the jamming technology requirements of the pods are noise jamming, deceptive jamming and complex jamming; the jamming ability requirement of the pods is adaptive and multitarget jamming.

\section{ESTABLISH JAMMING MODEL OF THE PODS}

In order to facilitate research, we respectively established the electromagnetic environment models, radar's detection models before and after jammed and the jamming tactical models to study the combat operation of the pods.

\section{A. Electromagnetic Environment Models}

The electromagnetic environment models include electromagnetic waves propagating model and frequency distribution of electromagnetic waves.
The propagating velocity of electromagnetic waves equals to the speed of light. Electromagnetic waves propagate in space, the directions of the nearest electric field or magnetic field are the same, the distance between adjacent peaks is the wavelength $(\lambda)$, and the number of vibrations per second is the frequency of electromagnetic waves (f). In vacuum, the velocity of the electromagnetic wave is equal to the speed of light. In the atmosphere, the propagation velocity of electromagnetic waves is less than the speed of light. The propagation speed of light in vacuum is $2.9979246 \times 10^{8} \mathrm{~m} / \mathrm{s}$, engineering generally takes $3 \times 10^{8} \mathrm{~m} / \mathrm{s}[3]$

Microwave's frequency is very high, and the wavelength is very short, so its ground wave attenuation soon. That leads to that we can't rely on the ground wave for more distant transmission. Ultra short waves, especially microwaves, are mainly propagated by spatial waves. Simply put it, a space wave is a wave that travels in a straight line in a spatial range. Obviously, because of the curvature of the earth, there is a limit to the direct distance traveled by space waves, it is Rmax. In the area of the farthest direct distance, it is customary to be called a lighting area; on the contrary, it is called shaded area.

This article supposed that the location between the pod and the ground radar suffices the sight transmission conditions of microwave, so we can use the attenuation equation of the electromagnetic wave to calculate the attenuation of the jamming power[6].

\section{B. Radar Detection Model before and after Jammed}

According to the radar equation, the maximum detection distance $R_{\max }(\theta, \varphi)$ of the radar at an azimuth and elevation is calculated by formula (1) when the radar is unjammed [1] [3].

$$
R_{\max }(\theta, \varphi)=\left[\frac{P_{t} \tau G_{t} G_{r} \sigma \lambda^{2} F_{t}^{2}(\theta, \varphi) F_{r}^{2}(\theta, \varphi)}{(4 \pi)^{3} k T_{s} V_{0} C_{B} L_{s}}\right]^{1 / 4}
$$

Where: $P_{t}$---- radar transmitter peak power, unit : watt;

$\tau$---- radar pulse width, unit : second(s);

$G_{t}{ }^{----}$radar's transmitting antenna gain;

$G_{r}{ }^{----}$radar's receiver antenna gain ;

$\sigma$---- radar's target scattering cross section, unit : square meter $\left(\mathrm{m}^{2}\right)$;

$\lambda$---- radar wavelength, unit : meter(m); 
$F_{t}^{2}(\theta, \varphi)$---- transmitting pattern factor of the radar antenna in the direction of the target;

$F_{r}^{2}(\theta, \varphi)$---- receiving pattern factor of the radar antenna in the direction of the target;

$k$---- Boltzmann constant, $k=1.38 \times 10^{-23} \mathrm{~W} \cdot \mathrm{s} / \mathrm{K}$;

$V_{0}$---- visibility factor, that is minimum detectable signal to noise ratio(SNR);

$C_{B}{ }^{----}$receiver's bandwidth correction factor, usually taken 1 ;

$L_{s}{ }^{----}$system's loss;

$T_{s}$---- radar receiver input equivalent noise temperature, unit : $\mathrm{K}$;

$T_{\mathrm{s}}$ usually calculated by the following formula:

$$
T_{s}=\left(0.876 T_{A}^{\prime}+36\right)+290\left(L_{r}-1\right)+290(N F-1) L_{r}
$$

Where: $T_{A}^{\prime}$---- noise temperature of the radar antenna;

$L_{r}$---- receive branch feeder loss;

$N F$---- equivalent noise figure of the system;

$T_{s}$ can also be directly equivalent to $T_{A}^{\prime}$ when calculated.

After the radar is jammed by blanket jamming, the jamming power spectral density $P_{j B}$ at the radar antenna is:

$$
P_{j B}=\frac{P_{j} G_{j}}{4 \pi R_{j}^{2}} \times \frac{1}{B_{j}} \times \frac{1}{L_{a j} L_{j}}
$$

Where: $P_{i}----$ jammer output power, unit : watt;

$G_{j}{ }^{----}$jammer's antenna gain;

$R_{j}{ }^{----}$the distance between the jammer and the radar, unit : meter(m);

$B_{j}{ }^{----}$jamming signal's bandwidth, unit : $\mathrm{Hz}$;

$L_{a j}$---- the one-way atmospheric transmission loss of the jamming signal at distance $R_{j}$, the near-range loss in the test can be approximated $0 \mathrm{~dB}$;

$L_{j}$---- the transmitter 's emission loss.

When there is no jamming, the noise power in the radar receiver is $P_{N}$. At blanket jamming environment, jamming power $P_{j c}$ which enter into the radar receiver is:

$$
P_{j c}=\frac{P_{j} G_{j}}{4 \pi R_{j}^{2}} \times \frac{1}{B_{j}} \times \frac{1}{L_{a j} L_{j}} \times \frac{G_{r} \lambda^{2} B_{n}}{4 \pi}
$$

Where: $B_{n}{ }^{----}$radar signal's bandwidth, unit : $\mathrm{Hz}$.

$P_{N}$ and $P_{j C}$ can be recorded in the test, or converted by the recorded noise level. The relationship between the receiver noise temperature and the noise power is known as follow:

$$
k T_{s}=P_{N} / B_{n}
$$

Then at the blanket jamming environment, the relationship between the noise temperature $T_{j}$ and the jamming power in the receiver is:

$$
k T_{j}=P_{j c} / B_{n}=\frac{P_{j} G_{j}}{\left(4 \pi R_{j}\right)^{2}} \times \frac{1}{B_{j}} \times \frac{G_{r j} \lambda^{2}}{L_{j} L_{a j}}
$$

Assuming that the parameters of the radar before and after jammed does not change, we can use above formulas to calculate the maximum detection distance of the radar when it is normal working or jammed[4] [5].

\section{Jamming's Tactical Model in Air to Ground Fight Mode}

When performing air-to-ground penetration tasks, the key electronic targets of the pod will also change depending on the electromagnetic threat received by the receiving system. After entering the ground-radar early warning and detection area, the pod needs to jam the radar's detection and tracking system of the ground-to-air missile. Its tactical diagram is shown in Fig. 1.

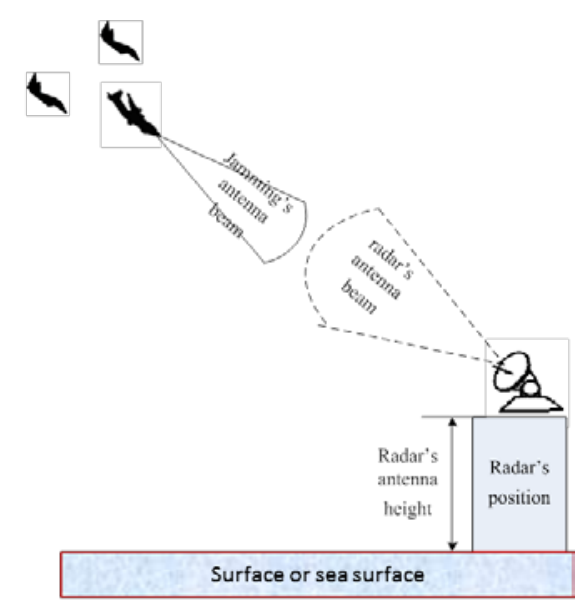

Fig. 1. Jamming model for air-to-ground penetration

If there are fighters or bombers formation flying, the carrier aircraft can spread jamming signals through the pod to reduce the detection distance of the enemy radar to achieve the purpose of protecting the flight formation. According to the aircraft flight height, air-to-ground penetration can be divided into high-altitude penetration and low-altitude or extreme lowaltitude penetration. This article mainly considers the latter.

\section{COMPUTER SIMULATION}

\section{A. Jamming Effect when Low-Altitude Penetration (flight height:1000m)}

\section{1) Single Aircraft Penetration}

When the target is in low-altitude flight, the clutter enter into the radar is less than the extreme low-altitude. At this set detection SNR is $10 \mathrm{~dB}$, the antenna efficiency is 0.9 , the antenna noise temperature is $1000 \mathrm{~K}$. The detection range of radar for a single plane is shown in Fig 2.

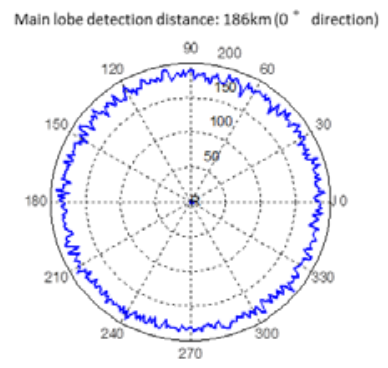

Fig. 2. Radar's Detection Distance Curve on a Single Aircraft without Jamming (low-altitude) 
It can be seen from Fig. 2 that the detection distance of the radar will change due to the influence of noise and clutter. However, the distance of the radar in the $360^{\circ}$ range is about $184 \mathrm{~km}$. The radar detection range in the $0^{\circ}$ direction is about $186 \mathrm{~km}$.

The distance of the radar is shown in Fig. 3 after the pod jammed it at different distances.

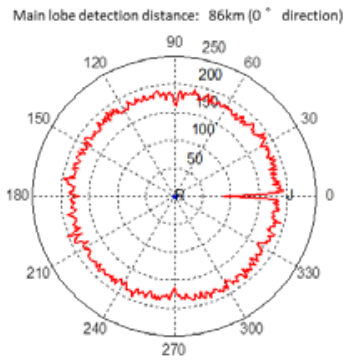

(a)range is $200 \mathrm{~km}$

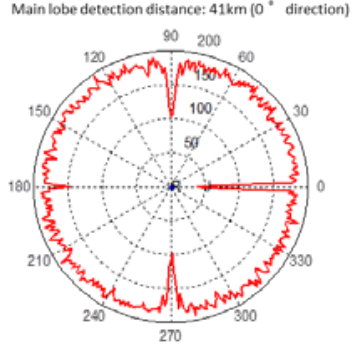

(c)range is $50 \mathrm{~km}$

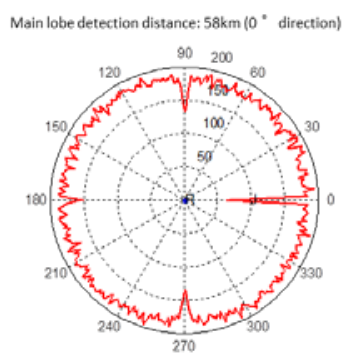

(b)range is $100 \mathrm{~km}$

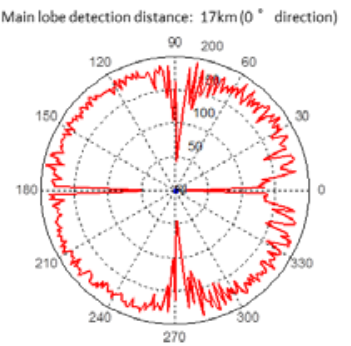

(d)range is $10 \mathrm{~km}$
Fig. 3. Radar's Detection Distance Curve on a Single Aircraft within Jamming

Compared with the radar detection distance curve of different distances in Fig. 3, it can be seen that the detection distance near the main lobe decreases as the loader continues to fly to the radar. The relationship between the distance of the radar and the distance of jamming is shown in table I.

TABLE I. DIFFERENT JAMMING DisTANCE, RADAR’s MAIN LOBE DETECT DISTANCE (LOW-ALTITUDE, SINGLE AIRCRAFT)

\begin{tabular}{|c|c|c|}
\hline $\begin{array}{c}\text { the distance between the } \\
\text { aircraft and the radar }\end{array}$ & $\begin{array}{c}\text { radar's main lobe } \\
\text { detect distance }\end{array}$ & $\begin{array}{c}\text { distance suppression } \\
\text { percentage }\end{array}$ \\
\hline 200 & 86 & $46 \%$ \\
\hline 100 & 58 & $31 \%$ \\
\hline 50 & 41 & $22 \%$ \\
\hline 10 & 17 & $9 \%$ \\
\hline
\end{tabular}

It can be seen from Fig. 3, 50km away, the radar's detection range is less than the distance between the aircraft and radar, that is, the radar can't detect the aircraft at this time. When the distance between the aircraft and the radar is less than $25 \mathrm{~km}$, the radar's detection distance is $17 \mathrm{~km}$, and the radar can detect the aircraft. After calculation, when the distance between the aircraft and the radar is $38 \mathrm{~km}$, the radar's detection distance is $38 \mathrm{~km}$. Therefore, at this time the radar's burn-through distance of $38 \mathrm{~km}$.

\section{2) Double Formation Penetration}

When two aircrafts formation penetrate, the RCS of the formation increase. But if the jamming pods of the formation jam the radar at the same time, the jamming power greatly increased.

When there is no jamming, the detection distance curve of the radar is shown in Fig 4.

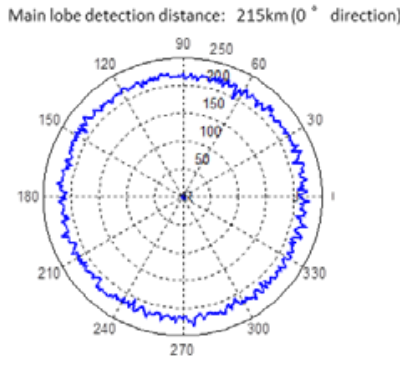

Fig. 4. Radar's Detection Range on the Formation without Jamming (low altitude)

When the formation jam the radar at the same time, the radar's detection range on the formation is shown in Fig 5.

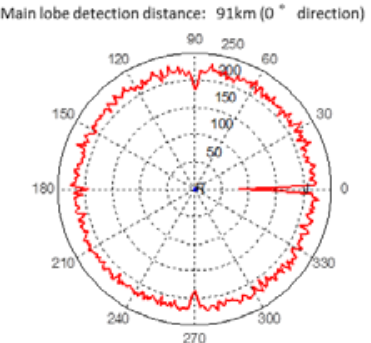

(a)range is $200 \mathrm{~km}$

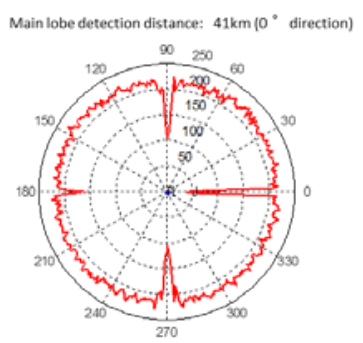

(c)range is $50 \mathrm{~km}$

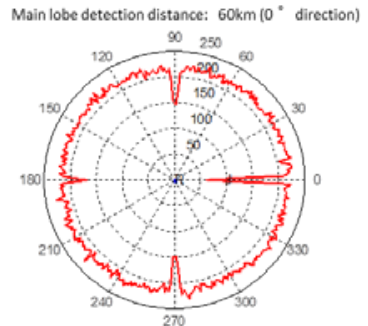

(b)range is $100 \mathrm{~km}$
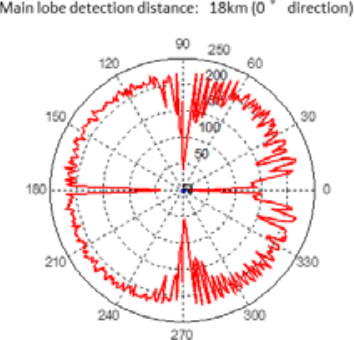

(d)range is $10 \mathrm{~km}$
Fig. 5. Radar's Detection Range on the Formation within Jamming (low altitude)

As it can be seen from Fig. 5, two pod's jamming effect of the formation roughly equivalent to the single aircraft's. As the formation increased RCS, the effect of power synthesis will be discounted, so the radar's detection distance increased slightly. The relationship between the detection distance of the radar and the jamming distance of the pod is shown in Table II . At this point, the radar's burn-through distance is $40 \mathrm{~km}$.

TABLE II. DIFFERENT JAMMING DisTANCE, RADAR'S MAIN LOBE DETECT DISTANCE (LOW-ALTITUDE, DOUBLE FORMATION)

\begin{tabular}{|c|c|c|}
\hline $\begin{array}{c}\text { the distance between the } \\
\text { aircraft and the radar }\end{array}$ & $\begin{array}{c}\text { radar's main lobe } \\
\text { detect distance }\end{array}$ & $\begin{array}{c}\text { distance suppression } \\
\text { percentage }\end{array}$ \\
\hline 200 & 91 & $42 \%$ \\
\hline 100 & 60 & $28 \%$ \\
\hline 50 & 41 & $19 \%$ \\
\hline 10 & 18 & $8 \%$ \\
\hline
\end{tabular}




\section{B. Jamming Effect when Extreme low-altitude Penetration (flight height 100m)}

When the target is flight in extreme low-altitude, the clutter enter into the radar is more than the low-altitude, radar requires a larger signal-to-noise ratio to detect the target. At this set detection SNR is $15 \mathrm{~dB}$, the antenna efficiency is 0.9 , the antenna noise temperature is $1500 \mathrm{~K}$. The detection range of radar for a single plane is shown in Fig 6 .

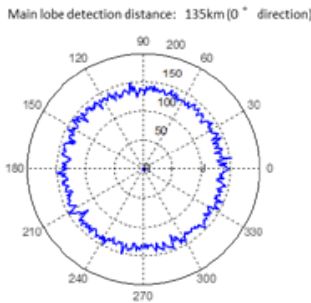

Fig. 6. Radar's Detection Distance on a Single Aircraft without Jamming (extreme low-altitude)

It can be seen from Fig. 6 that the detection distance of the radar is about $135 \mathrm{~km}$.

The distance of the radar is shown in Fig. 7 after the pod jammed it at different distances.

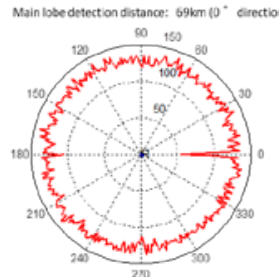

(a)range is $200 \mathrm{~km}$

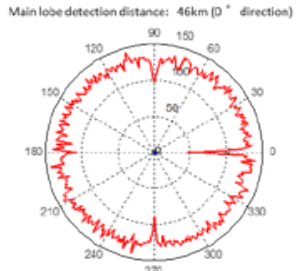

(b)range is $100 \mathrm{~km}$
Fig. 7. Radar's Detection Distance on a Single Aircraft within Jamming

As it can be seen from Fig. 7, when the pod is jamming at $200 \mathrm{~km}$, the main lobe of the radar's detection distance is reduced to about $69 \mathrm{~km}$. At this point, the radar's burn-through distance is about $19 \mathrm{~km}$.

In the case of a double-plane formation, it is assumed that they both in the same beam of the radar, but the RCS of the target increases, and the combined power of the jamming also increases. When the pod jamming at different distances, the detection distance of the radar without or within jamming are shown in Fig 8 and Fig 9.

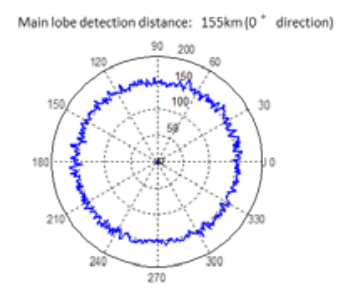

Fig. 8. Radar's Detection Range on the Formation without Jamming (extreme low-altitude)
As it can be seen from Fig. 9, when the pods of the formation are jamming at $200 \mathrm{~km}$, the main lobe of the radar's detection distance is reduced to about $75 \mathrm{~km}$, the radar's burnthrough distance is about $18 \mathrm{~km}$.
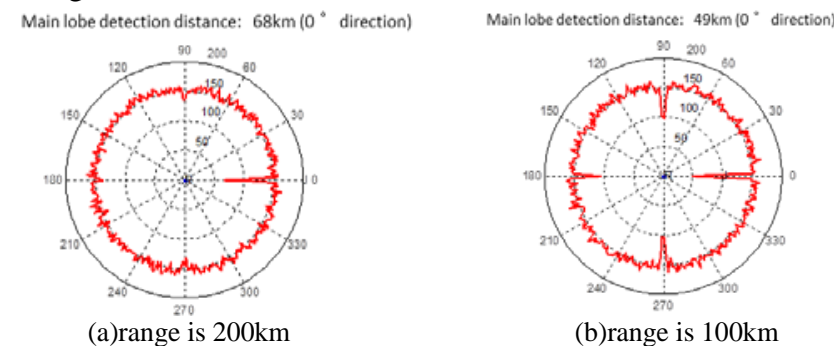

(b)range is $100 \mathrm{~km}$

Fig. 9. Radar's Detection Range on the Formation within Jamming (extreme low-altitude)

Compared to single and double planes, it can be seen that although the double-aircraft increases the RCS of the radar while increasing the radar's detection distance (without jamming), but within jamming, the radar's detection distance drops more. This is because synthesis power of the two pods obtaining greater jamming gain than a single pod.

From the above simulation, it can be seen that the jamming pod can effectively protect the aircraft close to the ground radar about $30 \mathrm{~km}$ distance, to achieve the purpose of penetration.

\section{CONCLUSION}

This article deals with the use of jamming pods, established the corresponding combat operational model, and simulated the effect of the jamming pods used in the air-to-ground penetration combat scene. Considered both low and extreme low-altitude situations, give the jamming results at different distances. The results show that in low-altitude and extreme low-altitude penetration, jamming pods can effectively reduce the detection range of ground-to-air defense radar, the lower the flight height, the greater the radar detection performance decline.

\section{REFERENCES}

[1] M I Skolink,Introduction to Radar Systems, third edition,McGram-Hill Book Co.2004,pp.66-100

[2] M I Skolink, Radar Handbook, McGram-Hill Book Co,1990,pp.53-89

[3] D K Barton, Radar System Analysis and Modeling, Artech House Inc,2005,pp.83-120

[4] Bassem R.Mahafza, Radar Systems Analysis and Design Using MATLAB,second edition,Chapman \&Hall/CRC,2005,pp.21-28

[5] D.Curtis Schleher, Electronic Warfare in the Information Age, Artech House, 1999

[6] Xiang Jingcheng, Radar System, Beijing:Publishing House of Electronics Industry, 2001 